\title{
SIRT6 suppresses glioma cell growth via induction of apoptosis, inhibition of oxidative stress and suppression of JAK2/STAT3 signaling pathway activation
}

\author{
JUN FENG, PENG-FEI YAN, HONG-YANG ZHAO, FANG-CHENG ZHANG, \\ WO-HUA ZHAO and MIN FENG \\ Department of Neurosurgery, Union Hospital, Tongji Medical College, \\ Huazhong University of Science and Technology, Wuhan, Hubei 430022, P.R. China
}

Received September 4, 2015; Accepted October 1, 2015

DOI: $10.3892 /$ or.2015.4477

\begin{abstract}
Sirtuin 6 (SIRT6) is a member of the mammalian $\mathrm{NAD}^{+}$-dependent deacetylase sirtuin family that acts to maintain genomic stability and to repress genes. SIRT6 has recently been reported to be a tumor suppressor that controls cancer metabolism, although this effect of SIRT6 is still in dispute. Moreover, the role of SIRT6 in glioma is largely unknown. In the present study, we found that overexpression of SIRT6 using an adenovirus inhibited glioma cell growth and induced marked cell injury in two glioma cell lines (U87-MG and T98G). Fluorescent terminal deoxyribonucleotidyl transferase (TdT)-mediated biotin-16-dUTP nick-end labelling (TUNEL) assay showed that SIRT6 overexpression induced obvious apoptosis in the T98G glioma cells. Immunoblotting and immunofluorescent staining demonstrated that SIRT6 overexpression promoted the mitochondrial-to-nuclear translocation of apoptosis-inducing factor (AIF), a potent apoptosis inducer. Moreover, we found that SIRT6 overexpression largely reduced oxidative stress and suppressed the activation of the JAK2/STAT3 signaling pathway in glioma cells. Finally, we showed that SIRT6 mRNA and protein levels in human glioblastoma multiforme tissues were significantly lower than the levels in peritumor tissues. In summary, our data suggest that SIRT6 suppresses glioma cell growth via induction of apoptosis, inhibition of oxidative stress and inhibition of the activation of the JAK2/STAT3 signaling pathway. These results indicate that SIRT6 may be a promising therapeutic target for glioma treatment.
\end{abstract}

Correspondence to: Dr Min Feng, Department of Neurosurgery, Union Hospital, Tongji Medical College, Huazhong University of Science and Technology, Wuhan, Hubei 430022, P.R. China E-mail: minfeng07@foxmail.com

Key words: SIRT6, glioma, apoptosis, oxidative stress, JAK2/STAT3

\section{Introduction}

Malignant glioma is a devastating neural neoplasm with no effective treatment. Glioblastoma multiforme (GBM) is one of the most common and aggressive malignant gliomas in adults and is associated with an extremely short survival time (<15 months) (1). Recent findings suggest that radiotherapy plus concomitant and adjuvant chemotherapy suppress the proliferation and tumorigenicity of human glioma cells and represent the standard of care for newly diagnosed GBM patients (1). However, GBM is difficult to cure due to the fact that the tumor cells cannot be completely removed surgically, and that metastatic GBM is rather resistant to radiotherapy and chemotherapy (1). This predicament reflects the poor understanding of the exact molecular mechanisms involved in the onset and pathogenesis of GBM. An important aspect of the pathogenesis of GBM lies in the malignant transformation resulting from the accumulation of genetic alterations and abnormal intracellular signaling pathways and growth factors (2-5). Aberrant proliferation of glioma cells is mediated by the combination of growth factors, including vascular endothelial growth factor, brain-derived neurotrophic factor, platelet-derived growth factor, hepatocyte growth factor and transforming growth factor- $\beta$ (TGF- $\beta$ ) $(6,7)$. These factors trigger downstream cascades of growth signaling pathways such as mammalian target of rapamycin (mTOR) (8), ERK1/2 (9), PI3K/AKT (10) and JAK2/STAT3 (11). In addition, when GBM recurs, it shows progression to a higher histologic grade with metastasis, a more complex situation (2). Thus, a further understanding of the mechanisms underlying the tumorigenesis of GBM is urgently needed.

The sirtuins are a conserved family of proteins possessing NAD-dependent deacetylase activity. Sirtuins are widely expressed in different tissues including the brain (12), and there are seven members of the sirtuin family in mammals (SIRT1-SIRT7) $(13,14)$. They are involved in promoting longevity, particularly longevity associated with calorie restriction (14-16). In addition, they act as cellular sensors to detect intracellular energy availability and modulate diverse biological functions, including lipid transport, insulin secretion, inflammation, oxidant stress and exercise and hypoxia (15-20). 
Importantly, they participate in tumorigenic processes such as epithelial-mesenchymal transition (21). Currently, activators of sirtuins have attracted much attention by eliciting multiple metabolic benefits that protect against diet-induced obesity, type 2 diabetes, and non-alcoholic fatty liver disease (14). In addition, several sirtuins have been reported to have roles in the central nerve system (CNS), including neuroprotection (22), neural differentiation (23) and neurogenesis (24).

SIRT6 is a nuclear histone lysine deacetylase (25). Similar to other sirtuins, SIRT6 participates in many intracellular events such as TNF- $\alpha$ secretion (26) and lipid transport (27). Interestingly, SIRT6 promotes resistance to DNA damage and suppresses genomic instability. Knockout of SIRT6 results in abnormalities that include lymphopenia, fat loss, cyrtosis, metabolic defects and eventually premature death (28). This DNA-repair activity of SIRT6 suggests its critical role in tumorigenesis. Indeed, Sebastian et al demonstrated that loss of SIRT6 leads to tumor formation, increased glycolysis and tumor growth (29). Moreover, loss of SIRT6 induces epigenetic changes that are relevant to hepatocellular carcinoma development in patients (30). These studies suggest that SIRT6 may be a tumor suppressor. However, there are conflicting results concerning this protein. High SIRT6 nuclear staining was found to be significantly associated with poorer overall survival in breast cancer (31). TGF- $\beta$-mediated hepatocellular carcinoma tumorigenicity was reported to be promoted by SIRT6 via suppression of cellular senescence (32). Thus, according to current information, SIRT6 actually participates in tumor biology, while the action of SIRT6 may be tumor type-dependent.

The role of SIRT6 in CNS tumors is still largely unknown. In the present study, we investigated the possible alteration of expression of SIRT6 in human GBM tissues. Moreover, we investigated whether SIRT6 can affect GBM cell growth and if so, to further study the underlying mechanisms.

\section{Materials and methods}

Reagents. Antibodies against phospho-JAK2, total-JAK2, phospho-STAT3 and total-STAT3 were purchased from Cell Signaling Biotechnology (Danvers, MA, USA). Antibodies against SIRT6, lamin A/C, apoptosis-inducing factor (AIF) and tubulin were purchased from Abcam (Cambridge, UK). Cell viability assay (MST-8) was purchased from Dojindo Molecular Technologies, Inc. (Kumamoto, Japan). Terminal deoxynucleotidyl transferase dUTP nick end labeling (TUNEL) kit and LDH leakage assay were purchased from Promega (Madison, WI, USA). Nuclear and mitochondrial protein isolation kits were purchased from Pierce (Rockford, IL, USA). Commercial kits for reactive oxygen species (ROS), malondialdehyde (MDA) and $\mathrm{CuZu} / \mathrm{MnSOD}$ activity were purchased from Beyotime Institute of Biotechnology (Jiangsu, China).

Glioma cell culture. Two human GBM cell lines (U87-MG and T98G) and one human normal glial cell line (HEB) used in the present study were purchased from the Cell Bank of the Institute of Biochemistry and Cell Biology, Shanghai Institutes for Biological Sciences. Cells were cultured in Dulbecco's modified Eagle's medium (DMEM) supplemented with $10 \%$ fetal bovine serum (FBS) in an incubator with $95 \% \mathrm{O}_{2}$ and $5 \% \mathrm{CO}_{2}$.

Cell viability assay. Cell viability was evaluated using a non-radioactive MST-8 assay as described previously (33). U87-MG and T98G cells $\left(5 \times 10^{3}\right)$ were transfected with Ad-GFP or Ad-SIRT6 and then cultured. On the day of measurement, $10 \mu \mathrm{l}$ of CCK-8 solution was added into the medium at 24,48 and $72 \mathrm{~h}$ for $1 \mathrm{~h}$ at $37^{\circ} \mathrm{C}$. The absorbance at $450 \mathrm{~nm}$ was recorded by a microplate reader, and the relative cell viability was calculated. Experiments were performed in duplicate.

Adenovirus construction and transfection. The adenoviruses were generated with the RAPAd ${ }^{\circledR} \mathrm{CMV}$ Adenoviral Bicistronic Expression System (Cell Biolabs, San Diego, CA, USA). We cloned the mouse SIRT6 cDNA and inserted it into pacAd5 CMV-IRES vector and linearized the vector by PacI. The purified linearized DNAs were cotransfected into 293 cells using Lipofectamine ${ }^{\mathrm{TM}}$ assay (Invitrogen) (33). The adenoviruses in the media and 293 cells were harvested 7 days post transfection. Three freeze/thaw cycles were applied to crush cells and release the viruses. The viruses were stored at $-80^{\circ} \mathrm{C}$. For viral transfection, $20 \mu \mathrm{l}$ of the virus was added into the culture medium ( $2 \mathrm{ml}$ ) for $6 \mathrm{~h}$. An adenovirus expressing GFP (Ad-GFP) was used as a control. Cells were then transfected with Ad-GFP or Ad-SIRT6 for $6 \mathrm{~h}$.

Lactate dehydrogenase $(L D H)$ assay. Cell injury was determined using the CytoTox-ONE LDH leakage assay as described previously $(22,34)$. In brief, the cell culture medium at different time points was transferred to a black fluorescence plate and incubated for $10 \mathrm{~min}$ with CytoTox-ONE reagent followed by stop solution. Fluorescence was measured at $560 / 590 \mathrm{~nm}$.

TUNEL. Fluorescent TUNEL staining in glioma cells was conducted as previously described $(35,36)$. At 4 days after transfection, the cells were incubated in TUNEL reaction solution for $2 \mathrm{~h}$ in the dark. The cells were then washed with phosphate-buffered saline (PBS) for three times. After washing, the cells were incubated with 4',6-diamidino-2-phenylindole (DAPI) counterstaining solution for $3 \mathrm{~min}$. The stained cells were assessed under a fluorescence microscope (IX81, Olympus). Apoptotic cells (TUNEL-positive, green) were counted. At least 10 visual fields were counted to calculate the proportion of apoptosis.

Oxidant stress levels. ROS, lipid peroxidation and $\mathrm{CuZu} / \mathrm{Mn}-\mathrm{SOD}$ activity were measured to evaluate the intracellular oxidant stress levels 4 days after transfection. ROS levels were measured as previously described (37). Lipid peroxidation and $\mathrm{CuZu} / \mathrm{MnSOD}$ activity were evaluated using MDA content as previously described (37).

Quantitative real-time PCR. Real-time PCR analysis was performed on OpticonDNA engine (MJ Research Inc.) as previously described (38). TRIzol (Invitrogen) was used to extract total RNA from the human GBM tissues. The samples (100 ng) were amplified using primers. Amplification primers 
A

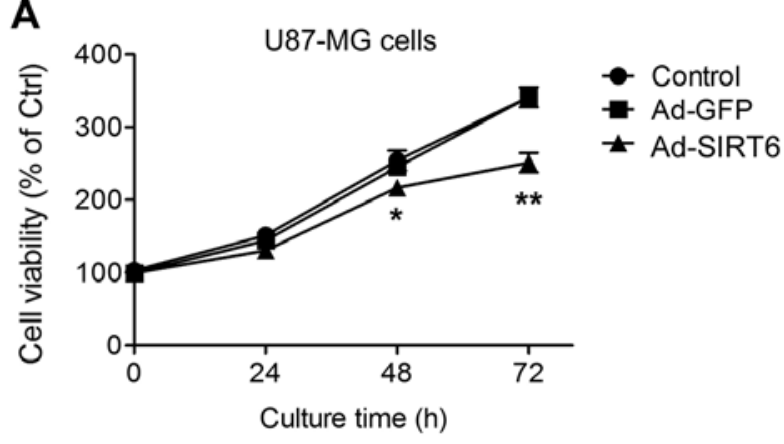

C

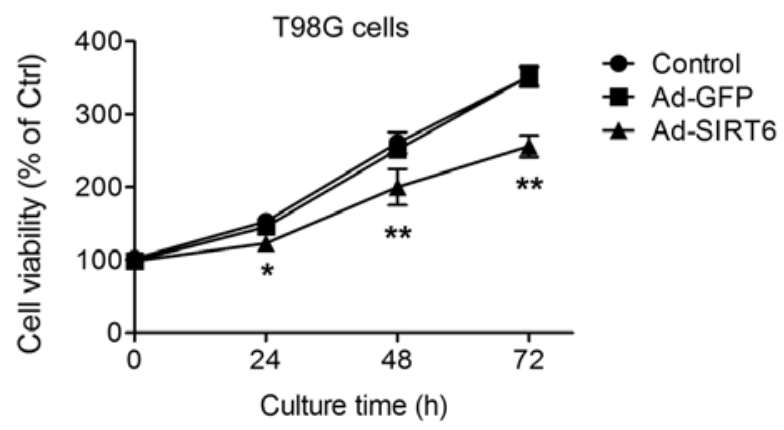

B

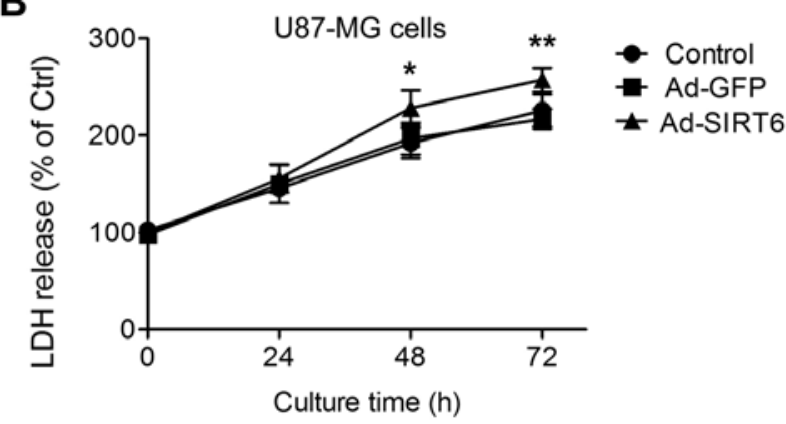

D

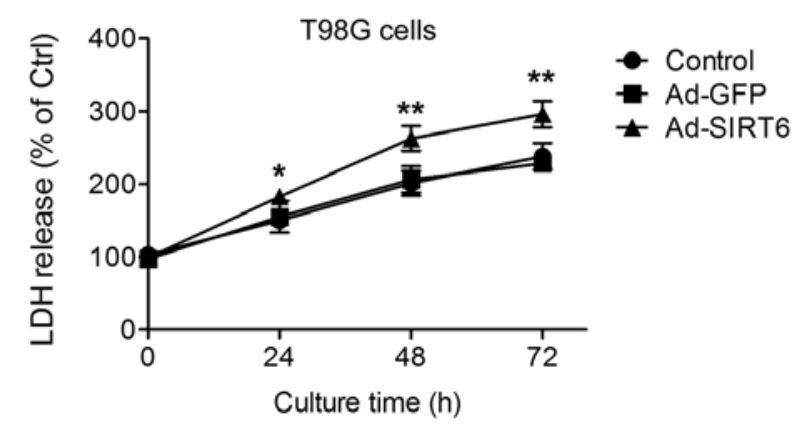

Figure 1. Glioma cell growth is inhibited by SIRT6 overexpression. (A and B) Cell viability assay and LDH release assay showing the influence of SIRT6 overexpression using an adenovirus on cell growth in U87-MG cells. ${ }^{*} \mathrm{P}<0.05,{ }^{* *} \mathrm{P}<0.01$ vs. Ad-GFP (vector control). $\mathrm{n}=8$. (C and $\left.\mathrm{D}\right) \mathrm{Cell}$ viability assay and LDH release assay showing the influence of SIRT6 overexpression using an adenovirus on cell growth in T98G cells. ${ }^{*} \mathrm{P}<0.05,{ }^{* *} \mathrm{P}<0.01 \mathrm{vs}$. Ad-GFP (vector control). $\mathrm{n}=8$.

for SIRT6 were purchased from GeneChem (Shanghai, China). The primers for $\beta$-actin are as follows: sense, GCACTCTTCC AGCCTTCCTTCC; antisense, CCGCCAGACAGCACTGT GTT. The mRNA level of housekeeping gene $\beta$-actin was used as a control.

Immunoblotting. Immunoblotting was performed as previously described (39). Cells were lysed using Triton X buffer (50 mM Tris, $150 \mathrm{mM} \mathrm{NaCl}, 1 \mathrm{mM}$ EDTA, 1\% Triton $\mathrm{X}$, $\mathrm{pH}$ 7.4) with protease inhibitors and boiled with SDS. Samples were run on $10 \%$ SDS-PAGE gel. Gels were transferred onto PVDF membranes and processed for immunoblotting with primary antibodies (SIRT6, 1:800; AIF, 1:1,000; lamin A/C, 1:1,000; p-JAK2, 1:500; t-JAK2, 1:500; p-STAT3, 1:500; t-STAT3, 1:500; tubulin, 1:1,000) and by corresponding HRP-labeled secondary antibodies. For immunoblotting, images were captured and processed with Odyssay (33).

Patients and tissue samples. A total of 4 patients (WHO grade II) enrolled in the present study underwent resection for GBM at the Department of Neurosurgery, Union Hospital, Tongji Medical College, Huazhong University, China. The tumor tissues and adjacent tissues (peritumoral) were rinsed in ice-cold PBS. The connective tissue or vessels were removed. The tissues were stored at $-80^{\circ} \mathrm{C}$. All of the tissue samples were re-evaluated according to WHO classifications (40). None of the patients had received chemotherapy or radiotherapy prior to surgery. The study was approved by the Institutional Review Board of Huazhong University and written informed consent was obtained from the patients or guardians.
Statistical analysis. Data are expressed as mean \pm SEM. Evaluation of the differences between groups was performed by two-samples Student's t-test or Mann-Whitney U test (non-normal distribution of values). $\mathrm{P}<0.05$ was considered to indicate a statistically significant difference.

\section{Results}

Overexpression of SIRT6 inhibits glioma cell growth. First, the influence of SIRT6 overexpression on GBM cell growth was assessed in vitro. Two human GBM cell lines (U87-MG and T98G) were used in the present study. In the U87-MG cells transfected with Ad-SIRT6, the cell growth was inhibited at 48 and $72 \mathrm{~h}$ after seeding (Fig. 1A). Accordingly, the LDH release into the culture medium of the SIRT6-overexpressing U87-MG cells was increased at 48 and $72 \mathrm{~h}$ (Fig. 1B). The cell viability and LDH release at the 24-h time-point were not significantly different among the cells (Fig. 1A and B). In the T98G cells, similar phenotypes were observed. Overexpression of SIRT6 inhibited T98G cell growth (Fig. 1C) and increased LDH release (Fig. 1D) at three time-points (24, 48 and $72 \mathrm{~h}$ ). These results suggest that overexpression of SIRT6 inhibits glioma cell growth and induces cell injury.

Overexpression of SIRT6 promotes apoptosis in glioma cells. As SIRT6 overexpression was able to inhibit glioma cell growth in the U87-MG and T98G cells, we investigated the molecular mechanisms of the tumor-suppressive effects of SIRT6 in the T98G cells in the following experiments. We performed TUNEL assay to examine the effect of SIRT6 


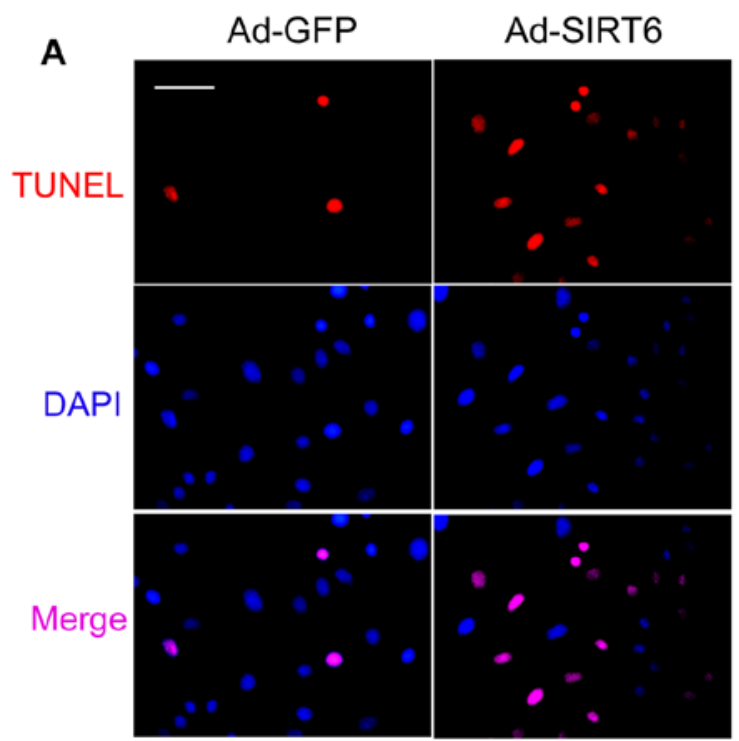

B

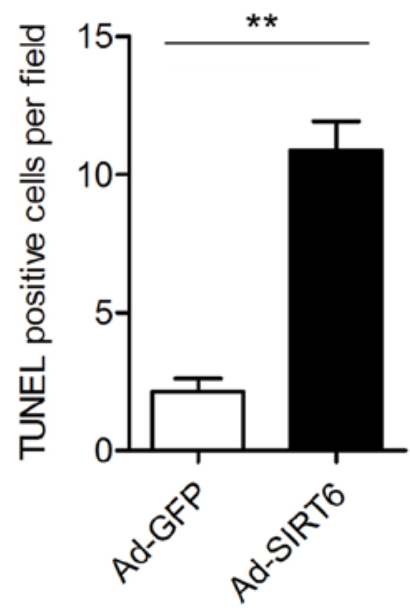

Figure 2. TUNEL staining of SIRT6-overexpressing glioma cells. Fluorescent images (A) and quantitative analysis (B) of TUNEL staining in T98G cells 4 days after transfection. DAPI was used to stain nuclei. ${ }^{* *} \mathrm{P}<0.01$ vs. Ad-GFP (vector control). $\mathrm{n}=6$. At least 30 visual fields were included for analysis.
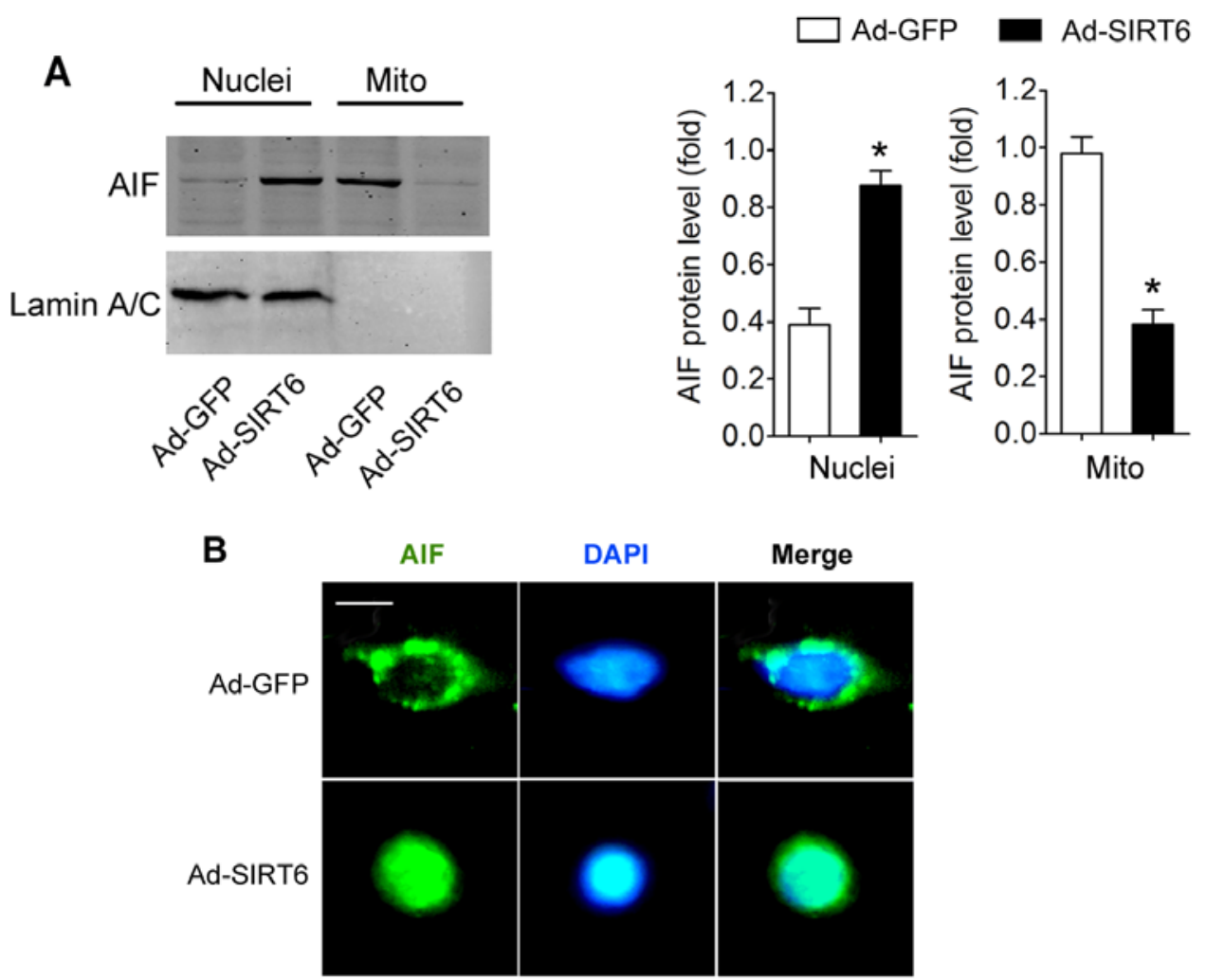

Figure 3. SIRT6 overexpression promotes AIF translocation in glioma cells. (A) Effects of SIRT6 overexpression on AIF mitochondrial-to-nuclear translocation 4 days after transfection. " $\mathrm{P}<0.05$ vs. Ad-GFP (vector control). $\mathrm{n}=4$. Mito, mitochondria. (B) Fluorescent staining showing that AIF entered nuclei in the SIRT6-overexpressing cells 4 days after transfection. DAPI was used to stain nuclei.

overexpression on the apoptosis of the T98G cells. There were fewer TUNEL-positive cells in the Ad-GFP group than the number in the Ad-SIRT6 group at 4 days after transfection (Fig. 2).

Overexpression of SIRT6 induces AIF mitochondrial-to-nuclear-translocation in the glioma cells.
Subsequently, we determined the potential effects of SIRT6 overexpression on AIF translocation. We found that the nuclear AIF expression in cells with SIRT6 overexpression was significantly higher than that in the Ad-GFP-transfected cells. Moreover, the mitochondrial AIF level in the cells with SIRT6 overexpression was reduced (Fig. 3A). Immunofluorescent staining of AIF clearly demonstrated 
A

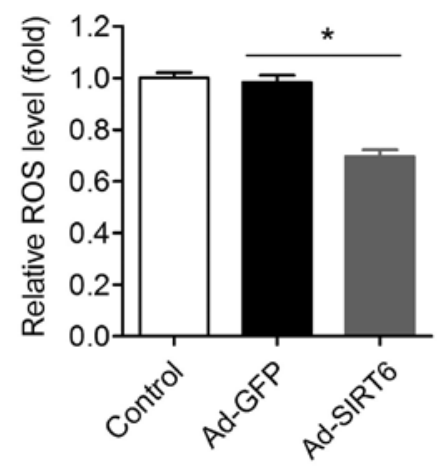

C

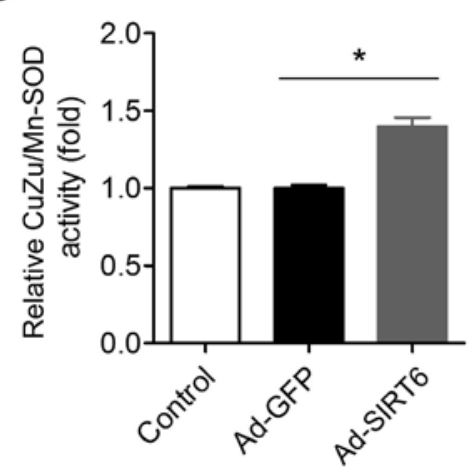

B

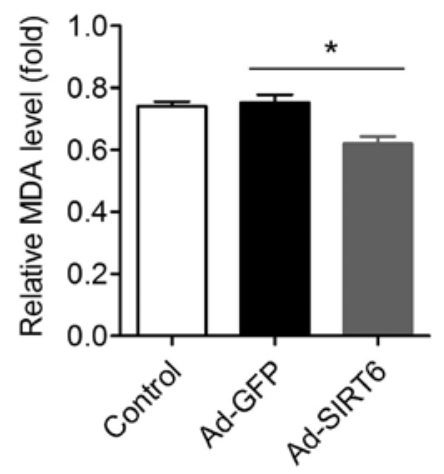

D

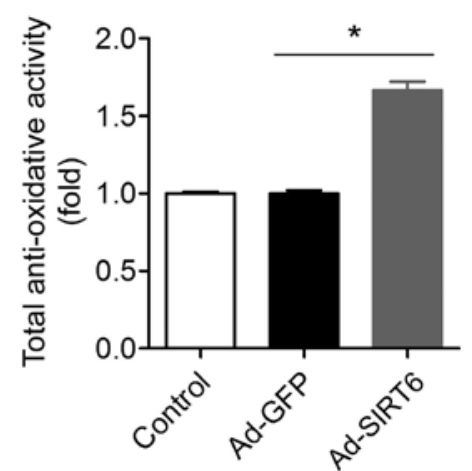

Figure 4. SIRT6 overexpression reduces intracellular oxidative stress in glioma cells. Relative ROS level (A), MDA level (B), CuZu/Mn-SOD activity (C) and total-oxidant activity (D) in Ad-GFP- or Ad-SIRT6-transfected T98G glioma cells. ${ }^{*} \mathrm{P}<0.05$ vs. Ad-GFP. $\mathrm{n}=8$.

A
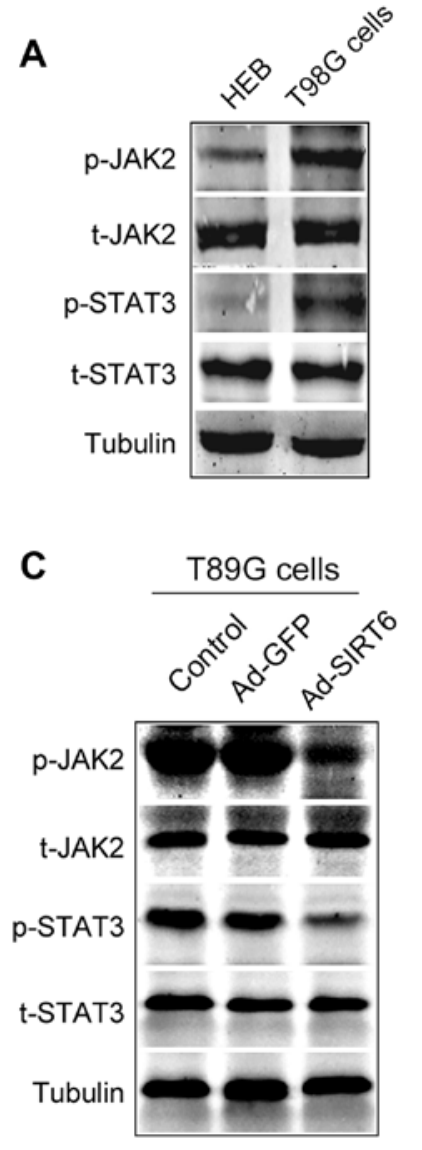

B
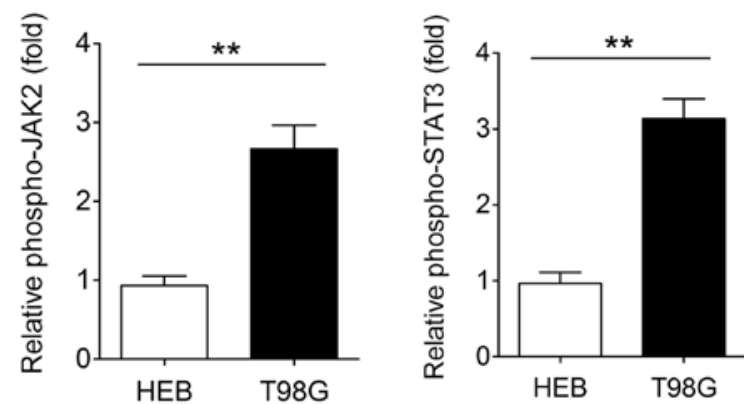

D
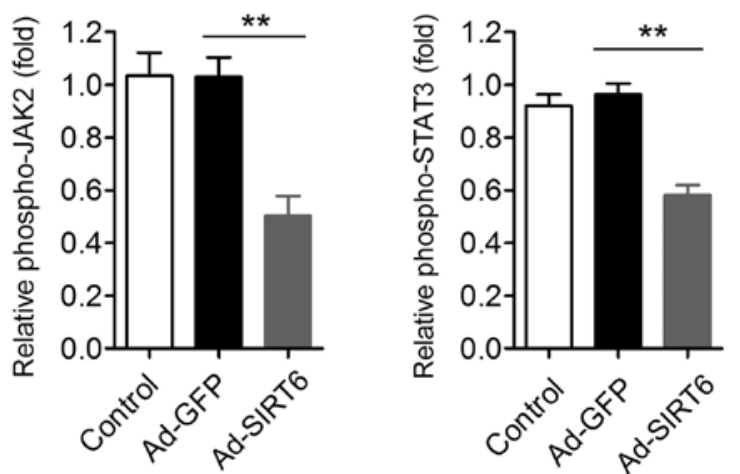

Figure 5. SIRT6 overexpression inhibits the JAK2/STAT3 signaling pathway in glioma cells. (A and B) Representative images of the phosphorylation of JAK2 and STAT3 in the (A) T89G and HEB cells and the (B) Ad-GFP- or Ad-SIRT6-transfected T98G glioma cells. (C and D) Quantitative analysis on the phosphorylation of JAK2 and STAT3 in the (C) T89G and HEB cells and the (D) Ad-GFP or Ad-SIRT6 transfected T98G glioma cells. ${ }^{* *}$ P $<0.01$ vs. Ad-GFP. $\mathrm{n}=6$. 
A
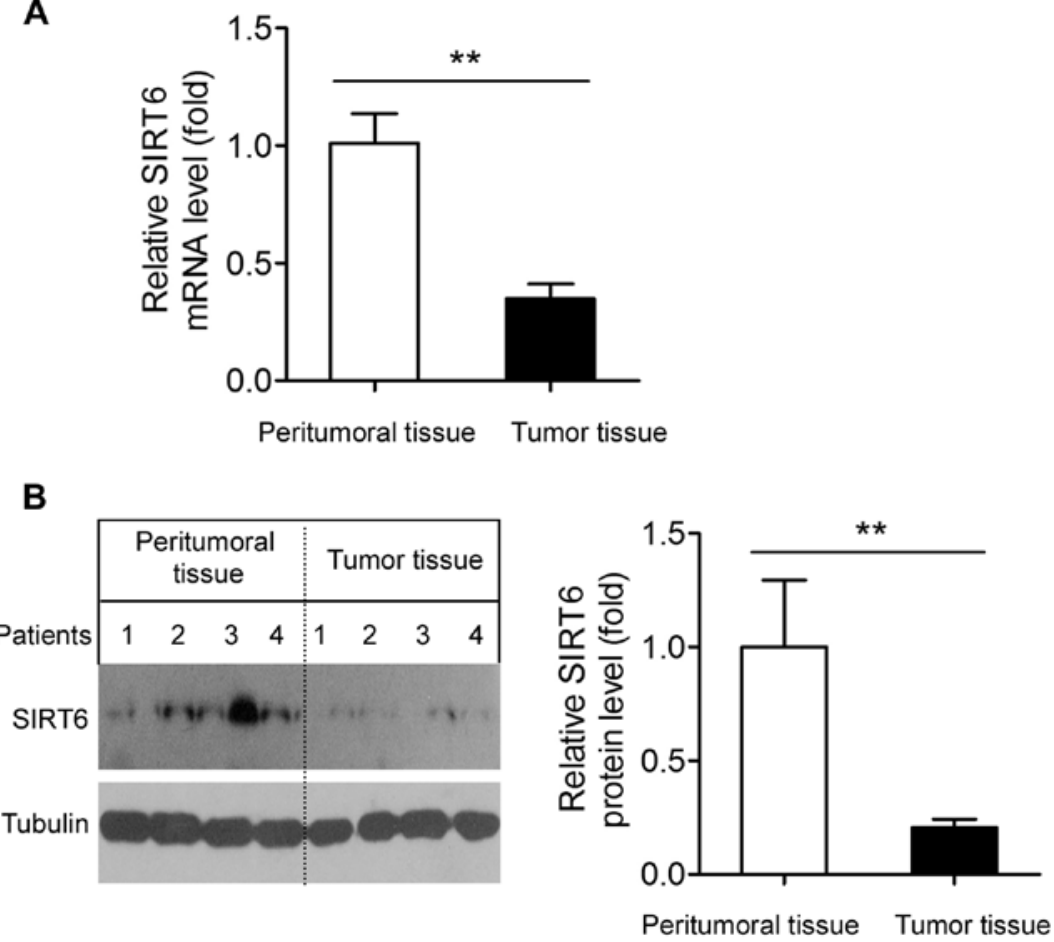

Figure 6. SIRT6 is downregulated in human primary GBM tissues. (A) Comparison of SIRT6 mRNA expression between GBM and peritumoral tissues. ${ }^{* *} \mathrm{P}<0.01$ vs. normal tissue. $\mathrm{n}=4$. (B) Comparison of SIRT6 protein expression between GBM and peritumoral tissues. ${ }^{* *} \mathrm{P}<0.01$ vs. normal tissue. $n=4$.

that AIF entered the nucleus in the cells with SIRT6 overexpression (Fig. 3B).

Oxidative stress in glioma cells is attenuated by SIRT6 overexpression. It is well-known that an abnormal increase in oxidative stress is an important factor for tumors (41). We compared the oxidant levels in SIRT6-overexpressing and control tumor cells. The ROS level in cells with SIRT1 overexpression was significantly lower than this level in the control cells (Fig. 4A). Moreover, the MDA level, an index for lipid peroxidation, was also reduced in the Ad-SIRT6-transfected cells (Fig. 4B). Accordingly, the $\mathrm{CuZu} / \mathrm{Mn}-\mathrm{SOD}$ activity in cells with SIRT1 overexpression was higher than that in the control cells (Fig. 4C). The total antioxidant activity in the SIRT6-overexpressed cells was also higher than that in the control cells (Fig. 4D).

Activation of the JAK2/STAT3 signaling pathway in glioma cells is suppressed by SIRT6 overexpression. The JAK2/STAT3 signaling pathway is constitutively activated in most primary malignant gliomas and the extent of activation is positively correlated with glioma grade (42). Thus, we determined the effect of SIRT6 overexpression on the JAK2/STAT3 signaling pathway. First, we confirmed that the JAK2/STAT3 signaling pathway was activated in the T98G cells compared with that in the HEB cells, a normal glial cell line (Fig. 5A and B). In addition, we found that the phosphorylation of JAK2 in the Ad-SIRT6transfected cells was significantly reduced compared with that in the Ad-GFP-transfected cells (Fig. 5C and D). The phosphorylation of STAT3 in the Ad-SIRT6-transfected cells was also decreased compared with that in the Ad-GFP-transfected cells (Fig. 5C and D). These data indicate that overexpres- sion of SIRT6 suppresses the activation of the JAK2/STAT3 signaling pathway in glioma cells.

SIRT6 is downregulated in human GBM tissues. Finally, we compared the SIRT6 mRNA and protein expression between peritumoral and tumor tissues in human samples. The SIRT6 mRNA level in the human GBM tissues was significantly $(\sim 65 \%)$ lower than that in the peritumoral tissues (Fig. 6A). Accordingly, SIRT6 protein expression in the GBM tissues was also shown to be significantly lower than that in the peritumoral tissues (Fig. 6B).

\section{Discussion}

In the present study, we showed that the growth of two GBM cell lines (U87-MG and T98G cells) was inhibited by SIRT6 overexpression. SIRT6 overexpression induced cell injury (LDH release). Moreover, we showed that SIRT6 overexpression not only led to apoptosis of the T98G cells, but also increased AIF mitochondrial-to-nuclear translocation. SIRT6 overexpression also inhibited the over-activated oxidative stress and JAK2/STAT3 signaling pathway in glioma cells. We also provide evidence that SIRT6 was markedly downregulated in human GBM tissues. These data collectively suggest that SIRT6 represents an anti-oncogene in glioma.

SIRT6 protein is expressed at the highest levels in the muscle, CNS and heart (43), and the cerebral SIRT6 expression was found to be reduced upon ischemic status (44). Notably, neural-specific SIRT6-knockout mice show attenuated somatic growth and obesity (45). In a recent report, Chen et al showed that SIRT6 is downregulated in human glioma tissues and glioma cell lines (46). They also showed that SIRT6 inhibits 
PCBP2 expression by deacetylating H3K9 (46). However, this study did not mention the type and WHO grade of the glioma tissues. In the present study, we assessed SIRT6 expression in human WHO grade II GBM tissues. GBM is the most common adult primary intracranial neoplasm and has a very poor outcome. In line with the results from Chen et al (46), we observed significant downregulation of SIRT6 in the GBM tissues. In addition, overexpression of SIRT6 suppressed the proliferation and induced cell injury in the cultured U87-MG and T98G cells. Since glioma tissue consistently displays a much higher ROS level (47), and SIRT6 overexpression reduces oxidant stress in glioma cells, we considered that the downregulation of SIRT6 in GBM tissues may contribute to the development of GBM by resulting in a pronounced increase in oxidative stress in GBM tissues to promote its growth.

SIRT6 suppresses genomic instability via binding chromatin and deacetylating histone $(25,28)$. Thus, maintenance of genomic integrity is the major function of SIRT6. Sebastián et al provided initial evidence that SIRT6 may be an anti-oncogene through regulating aerobic glycolysis in tumor cells (29). Marquardt et al showed that downregulation of SIRT6 and the dysregulated genes by loss of SIRT6 displayed oncogenic effects in hepatocarcinogenesis (30). These effects of SIRT6 strongly suggest a tumor-suppressing action, accompanied by modulation of histone deacetylation and glycolysis metabolism. We speculated that SIRT6 may also play an important role in cellular apoptosis. Indeed, we found that overexpression of SIRT6 induced pronounced apoptosis in glioma cells. According to our knowledge, the present study is the first to show the pro-apoptotic action of SIRT6 in glioma cells. This result is in agreement with a study conducted by Van Meter et al (48). They reported that SIRT6 overexpression induces massive apoptosis in cervical carcinoma, fibrosarcoma and breast tumor cell lines (48). However, SIRT6 overexpression has no effect on normal non-transformed cells (48). Intriguingly, they also found that the cell death induced by SIRT6 overexpression in cancer cells required the mono-ADP-ribosyltransferase activity of SIRT6 (48).

We observed that SIRT6 overexpression inhibited oxidative stress, evidenced by the suppressed ROS production and MDA level, and the increased CuZu/Mn-SOD activity and total antioxidant activity in the SIRT6-overexpressing glioma cells. Increased generation of ROS and an altered redox status have long been noted and considered as features of cancer cells (49). High grade glioma cells commonly display multiple genetic alterations and high oxidative stress, suggesting that the excessive redox state might be an important characteristic of invasive glioma (49). Moreover, the mutation of isocitrate dehydrogenase 1 (IDH1), a molecular basis of epigenomic changes in gliomas, is associated with excessive ROS production (50). In the light of these results, we proposed that the pro-apoptotic activity of SIRT6 in glioma cells may be attributed to the inhibitory action of SIRT6 on oxidative stress, at least in part. In addition, we found that SIRT6 overexpression suppressed the activation of the JAK2/STAT3 signaling pathway. The JAK2/STAT3 pathway was demonstrated to be highly activated in human GBM tissues and GBM-derived brain tumor stem cell-formed xenografts $(51,52)$. JAK2/STAT3-targeted therapy slowed the progression of GBM and suppressed glioma invasion $(51,52)$. Thus, abnormal JAK2/STAT3 activation is also a feature of GBM. As SIRT6 overexpression significantly inhibited JAK2/STAT3 activation in glioma cells, we believe that the inhibitory effect on the JAK2/STAT3 signaling pathway by SIRT6 may be another molecular mechanism underlying its tumor-suppressive effect.

Collectively, we demonstrated that SIRT6 overexpression inhibited cell growth, induced apoptosis and resulted in AIF mitochondrial-to-nuclear translocation in glioma cell lines. These phenotypes may be achieved by the reduced oxidative stress and JAK2/STAT3 activation under SIRT6 overexpression. Finally, we found that the expression of SIRT6 was lost in human GBM tissues. These results suggest that SIRT6 may be a promising therapeutic target for the treatment of malignant glioma.

\section{Acknowledgements}

The present study was supported by grants from the National Science Foundation of China (no. 81272778) and the National Science Foundation of Hubei, China (no. 2013CFB127).

\section{References}

1. Stupp R, Mason WP, van den Bent MJ, Weller M, Fisher B, Taphoorn MJ, Belanger K, Brandes AA, Marosi C, Bogdahn U, et al; European Organisation for Research and Treatment of Cancer Brain Tumor and Radiotherapy Groups; National Cancer Institute of Canada Clinical Trials Group: Radiotherapy plus concomitant and adjuvant temozolomide for glioblastoma. N Engl J Med 352: 987-996, 2005.

2. Alifieris $\mathrm{C}$ and Trafalis DT: Glioblastoma multiforme: Pathogenesis and treatment. Pharmacol Ther 152: 63-82, 2015.

3. Mair B, Kubicek S and Nijman SM: Exploiting epigenetic vulnerabilities for cancer therapeutics. Trends Pharmacol Sci 35: 136-145, 2014.

4. Huang M, Shen A, Ding J and Geng M: Molecularly targeted cancer therapy: Some lessons from the past decade. Trends Pharmacol Sci 35: 41-50, 2014.

5. Bouteldja N, Andersen LT, Møller N and Gormsen LC: Using positron emission tomography to study human ketone body metabolism: A review. Metabolism 63: 1375-1384, 2014.

6. Reardon DA and Wen PY: Glioma in 2014: Unravelling tumour heterogeneity-implications for therapy. Nat Rev Clin Oncol 12: 69-70, 2015.

7. Nicolaidis S: Biomarkers of glioblastoma multiforme. Metabolism 64 (Suppl 1): S22-S27, 2015.

8. Chiarini F, Evangelisti C, McCubrey JA and Martelli AM: Current treatment strategies for inhibiting mTOR in cancer. Trends Pharmacol Sci 36: 124-135, 2015.

9. Ji H, Wang J, Nika H, Hawke D, Keezer S, Ge Q, Fang B, Fang X, Fang D, Litchfield DW, et al: EGF-induced ERK activation promotes CK2-mediated disassociation of alpha-catenin from beta-catenin and transactivation of beta-catenin. Mol Cell 36: 547-559, 2009.

10. Miao H, Li DQ, Mukherjee A, Guo H, Petty A, Cutter J, Basilion JP, Sedor J, Wu J, Danielpour D, et al: EphA2 mediates ligand-dependent inhibition and ligand-independent promotion of cell migration and invasion via a reciprocal regulatory loop with Akt. Cancer Cell 16: 9-20, 2009.

11. Kim E, Kim M, Woo DH, Shin Y, Shin J, Chang N, Oh YT, Kim H, Rheey J, Nakano I, et al: Phosphorylation of EZH2 activates STAT3 signaling via STAT3 methylation and promotes tumorigenicity of glioblastoma stem-like cells. Cancer Cell 23: 839-852, 2013

12. Herskovits AZ and Guarente L: Sirtuin deacetylases in neurodegenerative diseases of aging. Cell Res 23: 746-758, 2013.

13. Haigis MC and Guarente LP: Mammalian sirtuins - emerging roles in physiology, aging, and calorie restriction. Genes Dev 20: 2913-2921, 2006.

14. Hubbard BP and Sinclair DA: Small molecule SIRT1 activators for the treatment of aging and age-related diseases. Trends Pharmacol Sci 35: 146-154, 2014. 
15. Polak-Jonkisz D, Rehan L, Laszki-Szczachor K and Sobieszczańska M: Novel targets for pharmacological intervention in age-related diseases. Trends Pharmacol Sci 35: 622-623, 2014

16. Imai $\mathrm{S}$ and Guarente $\mathrm{L}: \mathrm{NAD}^{+}$and sirtuins in aging and disease. Trends Cell Biol 24: 464-471, 2014.

17. Peng G and Liu Y: Hypoxia-inducible factors in cancer stem cells and inflammation. Trends Pharmacol Sci 36: 374-383, 2015.

18. Davis FM, Stewart TA, Thompson EW and Monteith GR Targeting EMT in cancer: Opportunities for pharmacological intervention. Trends Pharmacol Sci 35: 479-488, 2014.

19. Yang SJ and Lim Y: Resveratrol ameliorates hepatic metaflammation and inhibits NLRP3 inflammasome activation. Metabolism 63: 693-701, 2014.

20. Shimada T, Furuta H, Doi A, Ariyasu H, Kawashima H, Wakasaki H, Nishi M, Sasaki H and Akamizu T: Des-acyl ghrelin protects microvascular endothelial cells from oxidative stress-induced apoptosis through sirtuin 1 signaling pathway. Metabolism 63: 469-474, 2014

21. Chen J, Zhang B, Wong N, Lo AW, To KF, Chan AW, Ng MH, Ho CY, Cheng SH, Lai PB, et al: Sirtuin 1 is upregulated in a subset of hepatocellular carcinomas where it is essential for telomere maintenance and tumor cell growth. Cancer Res 71 : 4138-4149, 2011.

22. Wang P, Xu TY, Guan YF, Tian WW, Viollet B, Rui YC, Zhai QW Su DF and Miao CY: Nicotinamide phosphoribosyltransferase protects against ischemic stroke through SIRT1-dependent adenosine monophosphate-activated kinase pathway. Ann Neurol 69: 360-374, 2011

23. Prozorovski T, Schulze-Topphoff U, Glumm R, Baumgart J, Schröter F, Ninnemann O, Siegert E, Bendix I, Brüstle O, Nitsch R, et al: Sirt1 contributes critically to the redox-dependent fate of neural progenitors. Nat Cell Biol 10: 385-394, 2008.

24. Zhao Y, Guan YF, Zhou XM, Li GQ, Li ZY, Zhou CC, Wang P and Miao CY: Regenerative neurogenesis after ischemic stroke promoted by nicotinamide phosphoribosyltransferase-nicotinamide adenine dinucleotide cascade. Stroke 46: 1966-1974, 2015.

25. Michishita E, McCord RA, Berber E, Kioi M, Padilla-Nash H, Damian M, Cheung $\mathrm{P}$, Kusumoto $\mathrm{R}$, Kawahara TL, Barrett JC, et al: SIRT6 is a histone H3 lysine 9 deacetylase that modulates telomeric chromatin. Nature 452: 492-496, 2008.

26. Jiang H, Khan S, Wang Y, Charron G, He B, Sebastian C, Du J, Kim R, Ge E, Mostoslavsky R, et al: SIRT6 regulates TNF- $\alpha$ secretion through hydrolysis of long-chain fatty acyl lysine. Nature 496: 110-113, 2013.

27. Lee J, Hong SW, Park SE, Rhee EJ, Park CY, Oh KW, Park SW and Lee WY: Exendin-4 regulates lipid metabolism and fibroblast growth factor 21 in hepatic steatosis. Metabolism 63: 1041-1048, 2014.

28. Mostoslavsky R, Chua KF, Lombard DB, Pang WW, Fischer MR, Gellon L, Liu P, Mostoslavsky G, Franco S, Murphy MM, et al: Genomic instability and aging-like phenotype in the absence of mammalian SIRT6. Cell 124: 315-329, 2006.

29. Sebastián C, Zwaans BM, Silberman DM, Gymrek M, Goren A Zhong L, Ram O, Truelove J, Guimaraes AR, Toiber D, et al: The histone deacetylase SIRT6 is a tumor suppressor that controls cancer metabolism. Cell 151: 1185-1199, 2012.

30. Marquardt JU, Fischer K, Baus K, Kashyap A, Ma S Krupp M, Linke M, Teufel A, Zechner U, Strand D, et al: Sirtuin-6-dependent genetic and epigenetic alterations are associated with poor clinical outcome in hepatocellular carcinoma patients. Hepatology 58: 1054-1064, 2013

31. Khongkow M,Olmos Y,Gong C,Gomes AR,MonteiroLJ, YagüeE, Cavaco TB, Khongkow P, Man EP, Laohasinnarong S, et al: SIRT6 modulates paclitaxel and epirubicin resistance and survival in breast cancer. Carcinogenesis 34: 1476-1486, 2013.

32. Feng XX, Luo J, Liu M, Yan W, Zhou ZZ, Xia YJ, Tu W, Li PY, Feng ZH and Tian DA: Sirtuin 6 promotes transforming growth factor- $\beta 1 / \mathrm{H}_{2} \mathrm{O}_{2} / \mathrm{HOCl}$-mediated enhancement of hepatocellular carcinoma cell tumorigenicity by suppressing cellular senescence. Cancer Sci 106: 559-566, 2015.

33. Wang P, Xu TY, Guan YF, Su DF, Fan GR and Miao CY: Perivascular adipose tissue-derived visfatin is a vascular smooth muscle cell growth factor: Role of nicotinamide mononucleotide. Cardiovasc Res 81: 370-380, 2009.
34. Magzoub M and Miranker AD: Concentration-dependent transitions govern the subcellular localization of islet amyloid polypeptide. FASEB J 26:1228-1238, 2012.

35. Wang P, Guan YF, Du H, Zhai QW, Su DF and Miao CY: Induction of autophagy contributes to the neuroprotection of nicotinamide phosphoribosyltransferase in cerebral ischemia. Autophagy 8: 77-87, 2012.

36. Tönjes M,Barbus S, Park YJ, Wang W, Schlotter M, Lindroth AM, Pleier SV, Bai AH, Karra D, Piro RM, et al: BCAT1 promotes cell proliferation through amino acid catabolism in gliomas carrying wild-type IDH1. Nat Med 19: 901-908, 2013.

37. Song J, Ke SF, Zhou CC, Zhang SL, Guan YF, Xu TY, Sheng CQ, Wang P and Miao CY: Nicotinamide phosphoribosyltransferase is required for the calorie restriction-mediated improvements in oxidative stress, mitochondrial biogenesis, and metabolic adaptation. J Gerontol A Biol Sci Med Sci 69: 44-57, 2014.

38. Wang P, Du H, Zhou CC, Song J, Liu X, Cao X, Mehta JL, Shi Y, Su DF and Miao CY: Intracellular NAMPT-NAD ${ }^{+}-S I R T 1$ cascade improves post-ischaemic vascular repair by modulating Notch signalling in endothelial progenitors. Cardiovasc Res 104 477-488, 2014

39. Wang P, Xu TY, Wei K, Guan YF, Wang X, Xu H, Su DF, Pei G and Miao CY: ARRB1/ $\beta$-arrestin-1 mediates neuroprotection through coordination of BECN1-dependent autophagy in cerebral ischemia. Autophagy 10: 1535-1548, 2014.

40. Rousseau A, Mokhtari K and Duyckaerts C: The 2007 WHO classification of tumors of the central nervous system - what has changed? Curr Opin Neurol 21: 720-727, 2008.

41. Gorrini C, Harris IS and Mak TW: Modulation of oxidative stress as an anticancer strategy. Nat Rev Drug Discov 12: 931-947, 2013.

42. Lo HW, Cao X, Zhu H and Ali-Osman F: Constitutively activated STAT3 frequently coexpresses with epidermal growth factor receptor in high-grade gliomas and targeting STAT3 sensitizes them to Iressa and alkylators. Clin Cancer Res 14: 6042-6054, 2008.

43. Liszt G, Ford E, Kurtev M and Guarente L: Mouse Sir2 homolog SIRT6 is a nuclear ADP-ribosyltransferase. J Biol Chem 280: 21313-21320, 2005

44. Lee OH, Kim J, Kim JM, Lee H, Kim EH, Bae SK, Choi Y, Nam HS and Heo JH: Decreased expression of sirtuin 6 is associated with release of high mobility group box-1 after cerebral ischemia. Biochem Biophys Res Commun 438: 388-394, 2013.

45. Schwer B, Schumacher B, Lombard DB, Xiao C, Kurtev MV, Gao J, Schneider JI, Chai H, Bronson RT, Tsai LH, et al: Neural sirtuin 6 (Sirt6) ablation attenuates somatic growth and causes obesity. Proc Natl Acad Sci USA 107: 21790-21794, 2010.

46. Chen X, Hao B, Liu Y, Dai D, Han G, Li Y, Wu X, Zhou X, Yue Z, Wang L, et al: The histone deacetylase SIRT6 suppresses the expression of the RNA-binding protein PCBP2 in glioma. Biochem Biophys Res Commun 446: 364-369, 2014.

47. Santandreu FM, Brell M, Gene AH, Guevara R, Oliver J, Couce ME and Roca P: Differences in mitochondrial function and antioxidant systems between regions of human glioma. Cell Physiol Biochem 22: 757-768, 2008.

48. Van Meter M, Mao Z, Gorbunova V and Seluanov A: SIRT6 overexpression induces massive apoptosis in cancer cells but not in normal cells. Cell Cycle 10: 3153-3158, 2011.

49. Trachootham D, Alexandre J and Huang P: Targeting cancer cells by ROS-mediated mechanisms: A radical therapeutic approach? Nat Rev Drug Discov 8: 579-591, 2009.

50. Shi J, Sun B, Shi W, Zuo H, Cui D, Ni L and Chen J: Decreasing GSH and increasing ROS in chemosensitivity gliomas with IDH1 mutation. Tumour Biol 36: 655-662, 2015.

51. Stechishin OD, Luchman HA, Ruan Y, Blough MD, Nguyen SA, Kelly JJ, Cairncross JG and Weiss S: On-target JAK2/STAT3 inhibition slows disease progression in orthotopic xenografts of human glioblastoma brain tumor stem cells. Neuro Oncol 15: 198-207, 2013.

52. Zheng Q, Han L, Dong Y, Tian J, Huang W, Liu Z, Jia X, Jiang T, Zhang J, Li X, et al: JAK2/STAT3 targeted therapy suppresses tumor invasion via disruption of the EGFRvIII/JAK2/STAT3 axis and associated focal adhesion in EGFRvIII-expressing glioblastoma. Neuro Oncol 16: 1229-1243, 2014. 